\title{
Revisión
}

\section{Papel del estrés oxidativo en la esquizofrenia}

\author{
(The role of oxidative stress in schizophrenia)
}

María Clara Soto-Bernardini, Henriette Raventós-Vorst

\section{Resumen}

Centro de Investigación en Biología Celular y Molecular (CIBCM), Universidad de Costa Rica (UCR).

Abreviaturas: CAT, catalasa; $\mathrm{Cu}, \mathrm{Zn}-\mathrm{SOD}$, cobre, zincsuperóxido dismutasa; GCL, glutamato cisteína ligasa; GCLC, subunidad catalítica de glutamato cisteína ligasa; GCLM, modificador de glutamato cisteína ligasa; GHS, glutatión; GSS, glutatión sintetasa; GSHPx, glutatión peroxidasa; $\mathrm{H}_{2} \mathrm{O}_{2}$, peróxido de hidrógeno; MnSOD, manganeso superóxido dismutasa; MsrA, metionina sulfóxido reductasa $A$; $M s r B$, metionina sulfóxido reductasa $\mathrm{B}$; NO, óxido nítrico; $\mathrm{OH}$, ion hidroxilo; $\mathrm{O}_{2}$; superóxido; $\mathrm{ROS}$, especies de oxígeno reactivo; SOD, superóxido dismutasa; TEC, tratamiento electro convulsivo; $\mathrm{XO}, \quad$ xantinas oxidasas.

\section{Correspondencia:}

Henriette Raventós.

Centro de Investigación en Biología Celular y Molecular (CIBCM), Universidad de Costa Rica.

ISSN 0001-6002/2008/50/4/197-202 Acta Médica Costarricense, (O2008 Colegio de Médicos y Cirujanos
La esquizofrenia es una enfermedad mental severa que se presenta en alrededor del $1 \%$ de la población mundial. Para que se manifieste son necesarios tanto factores genéticos como ambientales, por lo que el genotipo de riesgo no conlleva al desarrollo de la enfermedad sino a una mayor susceptibilidad de presentarla. Existe evidencia de que el balance entre la producción de radicales libres y el sistema de defensa antioxidante está alterado en pacientes con esquizofrenia. Sin embargo, no se sabe con certeza si la producción de RLs tiene un papel causal en el desarrollo de la enfermedad o es producido durante el curso de la enfermedad, o por el uso de neurolépticos. En este artículo se revisa la evidencia de la participación de estas sustancias en el desarrollo de la enfermedad.

Descriptores: estrés oxidativo, esquizofrenia, oxi-radicales, sistema de defensa antioxidante

\section{Abstract}

Schizophrenia is a severe mental disorder that affects approximately $1 \%$ of the population worldwide. Genetic and environmental factors are important in its etiology, so the risk genotype increases the susceptibility to manifest the disorder. There is evidence that the balance between the production of free radicals and the antioxidant defense system is abnormal in schizophrenia patients. Nonetheless, it is not known if it plays a causal role in the development of the disorder, or if it is produced during the course of the disease, or as a consequence of antipsychotic treatment. In this paper, we review the evidence of the participation of these substances in the development of the illness.

Key words: oxidative stress, schizophrenia, oxyradicals, antioxidant defense system.

Recibido: 25 de octubre de $2007 \quad$ Aceptado: 12 de diciembre de 2007

La esquizofrenia es una enfermedad mental severa que se presenta en alrededor del $1 \%$ de la población mundial. ${ }^{1}$ En Costa Rica corresponde a una de las enfermedades psiquiátricas más costosas. En 2007, egresaron del Hospital Nacional Psiquiátrico (HNP) 1218 pacientes con esquizofrenia (50,3\% de ese total) y otros trastornos psicóticos.

Esto representó un total de estancias de 89247 días (77,1\% esquizofrenia) y cada día de internamiento tiene un costo de 42000 colones, sin incluir alimentación, medicamentos, laboratorio y tratamiento electro convulsivo(TEC). Por lo tanto, esto significó un costo de 3748 
374000 colones para el Sistema de Salud (departamento de registros médicos, HNP). Aunque no se cuenta con datos sobre la prevalencia de la esquizofrenia en el país, se cree que no es diferente al porcentaje arriba mencionado.

Esta enfermedad se caracteriza por la presencia de síntomas positivos (alucinaciones, delirios, comportamiento y lenguaje desorganizados) y negativos (aplanamiento afectivo, alogia y abulia). ${ }^{2} \mathrm{Al}$ igual que muchos desórdenes psiquiátricos, está clasificada como una enfermedad compleja, lo que significa que su origen no se puede atribuir a un solo gen o componente ambiental. ${ }^{1}$

Los familiares en primer grado de una persona con esquizofrenia tienen un riesgo 10 veces mayor de manifestar la enfermedad. La probabilidad en los padres es menor (alrededor del 6\%), posiblemente debido a que la esquizofrenia disminuye la posibilidad de tener hijos. Los parientes en segundo y tercer grado también muestran un riesgo un poco aumentado. ${ }^{3}$ La concordancia en gemelos monocigóticos es del 41-65\%, comparada con una del 0$28 \%$ en gemelos dicigóticos y se ha estimado una heredabilidad de alrededor del $85 \% .{ }^{4}$

Entre los factores ambientales relacionados con la esquizofrenia están las complicaciones obstétricas pre- y perinatales, como: bajo peso al nacer, incompatibilidad Rh, hipoxia, deficiencias nutricionales de la madre en el primer trimestre del embarazo, exposición al virus de la influenza en el segundo trimestre, estrés en la madre. ${ }^{5-6}$ Otras características como: nacimientos en invierno, lo cual puede estar relacionado con infecciones virales; ${ }^{6}$ residencia en zonas urbanas, y abuso de sustancias como anfetaminas, cocaína, halucinógenos ${ }^{5}$ y marihuana durante la adolescencia también, han sido asociadas. ${ }^{7}$

A pesar de las dificultades para replicar los resultados de otros estudios, debidas a los distintos factores ambientales, la participación de varios genes de efecto menor y la heterogeneidad genética, diversos estudios genómicos han encontrado algunos patrones consistentes. ${ }^{8}$ Dos meta análisis independientes, obtuvieron resultados significativos para las regiones $8 \mathrm{p}$ y $22 \mathrm{q} .{ }^{9-10}$

Una de las primeras teorías sobre la esquizofrenia propuso alteraciones en la neurotransmisión. Debido a esto, muchos de los estudios dirigidos a genes específicos que podrían conferir susceptibilidad para el desarrollo de esta enfermedad, se han enfocado en genes involucrados en los sistemas dopaminérgico, serotoninérgico y glutaminérgico. También han sido estudiados otros genes como el de la Neuregulina 1 (8p), Notch homolog 4 (6p) y Disbindina (6p). ${ }^{6}$ Otra de las teorías vinculada con la esquizofrenia involucra al estrés oxidativo. Sin embargo, no se conoce si este interviene como una causa primaria en el desarrollo de la esquizofrenia, o si es un desbalance producido durante el curso de la enfermedad, o como complicación por el uso de neurolépticos. ${ }^{11}$

\section{Radicales libres y sistema de defensa antioxidante}

Las especies de oxígeno reactivo (ROS), (Cuadro 1), sobre todo los oxi-radicales, son especies químicas que contienen oxígeno y son capaces de robar un electrón a moléculas orgánicas, inorgánicas y a átomos. Son generadas en condiciones fisiológicas durante el metabolismo aeróbico. $^{12}$ Estos radicales libres cumplen funciones fisiológicas y patológicas en el desarrollo celular, diferenciación y envejecimiento, y es el resultado de un balance entre la producción de estos y el sistema de defensa antioxidante. ${ }^{13}$

En el cerebro existe una alta demanda de oxígeno para mantener la fosforilación oxidativa y generar la energía requerida para las funciones neuronales. Alrededor del $90 \%$ del oxígeno celular sirve como aceptor de electrones en la fosforilación oxidativa. Sin embargo, algunos electrones pueden escapar de la cadena de transporte de electrones y reaccionar con oxígeno molecular, ${ }^{11}$ lo que conduce a la generación de oxi-radicales que pueden causar peroxidación de lípidos en la membrana mitocondrial. ${ }^{14}$ Estos pueden ser originados también por la acción de oxidasas (amino ácido oxidasas, citocromo oxidasas, monoaminas oxidasas, xantinas oxidasas), autoxidación de metales de transición (principalmente hierro y cobre) en presencia de oxígeno molecular y por oxidaciones fotoquímicas. ${ }^{11}$ Las membranas neuronales, al igual que las mitocondriales, son muy vulnerables a la peroxidación de lípidos. ${ }^{14}$

El sistema de defensa antioxidante incluye enzimas antioxidantes como la superóxido dismutasa (SOD), glutatión peroxidasa (GSHPx) y catalasa (CAT) (Figura 1). Además, comprende compuestos no enzimáticos como la albúmina, ácido úrico, bilirrubina, ácido ascórbico (vitamina C), $\alpha$ tocoferol (vitamina E), $\beta$-caroteno y glutatión (GSH). ${ }^{14-15}$

El óxido nítrico (NO) posee una función dual, ya que es un radical libre fisiológico, altamente reactivo, y puede generar especies de nitrógeno reactivo que son neurotóxicas para el sistema nervioso central. Por otro lado, el NO parece tener un efecto neuroprotector, pues reduce la generación de especies de oxígeno reactivas. En el cerebro el NO puede ser generado a partir de la sintasa óxido nítrica neural, o por la epitelial. $^{16}$

Otras enzimas involucradas en la reparación del daño oxidativo son las metionina sulfóxido reductasas (Msr). Las

Cuadro 1. Especies de oxígeno reactivo (ROS).

\begin{tabular}{|ll|ll|}
\hline \multicolumn{2}{|l|}{ Radicales libres } & No radicales \\
\hline Superóxido & $\mathrm{O}_{2}^{-}$ & Oxígeno molecular & ${ }^{1} \mathrm{O}_{2}$ \\
ion hidroxilo & $\mathrm{OH}$ & Peróxido de hidrógeno & $\mathrm{H}_{2} \mathrm{O}_{2}$ \\
Óxido nítrico & $\mathrm{NO}$ & Peroxinitrito & $\mathrm{NO}_{3}^{-}$ \\
\hline
\end{tabular}


ROS pueden oxidar los residuos de metionina en las proteínas a sulfóxido de metionona. Esto conlleva a la formación de dos formas epiméricas diferentes, metionina S-sulfóxido y metionina R-sulfóxido, que son reducidas por las proteínas MsrA y MsrB, respectivamente. ${ }^{17}$ Además de su papel en la reparación, la familia Msr contribuye a regular las funciones de proteínas por medio de la oxidación/reducción de residuos de metionina críticos y al mantenimiento del estado redox celular. $^{13}$

Cuando se genera un exceso de radicales libres o el sistema de defensa antioxidante es deficiente, los radicales pueden interactuar con lípidos, ácidos nucleicos y proteínas, causando daño e incluso muerte celular. ${ }^{12}$ Los productos de estas reacciones son utilizados como índices del daño oxidativo, tal es el caso de los ácidos grasos polinsaturados, mayores componentes de fosfolípidos de membrana y cuyos niveles se utilizan para medir el daño peroxidativo. ${ }^{14}$

El cerebro es más sensible al ataque de ROS que otros órganos, debido a su alto consumo de oxígeno, bajos niveles de enzimas antioxidantes, altos niveles de hierro y ascorbato (la mezcla de Fe/ascorbato puede ser un potente prooxidante para las membranas del cerebro) y alto contenido de ácidos grasos. ${ }^{18}$

\section{Evidencia de daño oxidativo y esquizofrenia}

Existe evidencia de que el balance entre la producción de radicales libres y el sistema de defensa antioxidante está alterado en la esquizofrenia. ${ }^{11}$ En 1954, se propuso por primera vez que los radicales tóxicos están involucrados en la etiología de la esquizofrenia. ${ }^{19}$

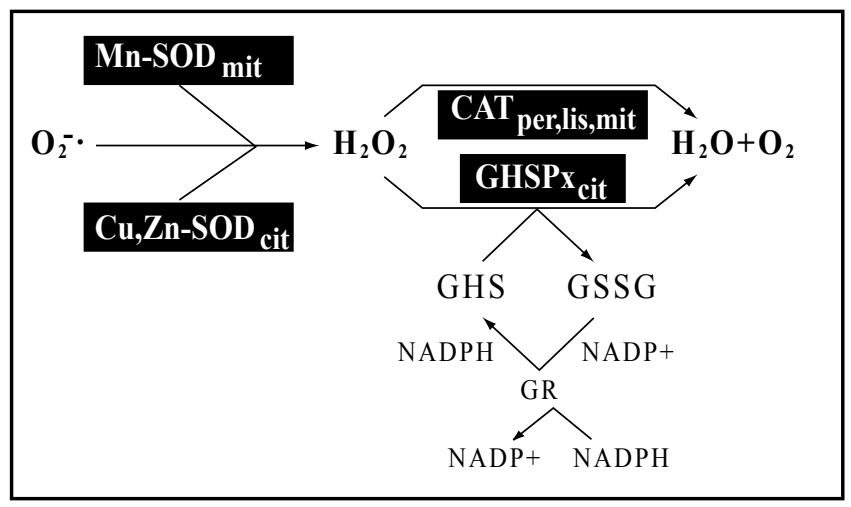

Figura 1. Sistema de defensa antioxidante. La superóxido dismutasa (SOD), ampliamente distribuida en cerebro, convierte el superóxido $\left(\mathrm{O}_{2}^{-}\right)$en peróxido de hidrógeno $\left(\mathrm{H}_{2} \mathrm{O}_{2}\right)$. Existen dos tipos de SODs: las cobre, zinc-superóxido dismutasas (Cu,Zn-SOD), localizadas predominantemente en el citosol y las manganeso superóxido dismutasas (Mn-SOD), en mitocondria. $\mathrm{El}_{2} \mathrm{O}_{2}$ es eliminado por la catalasa (CAT) (localizada principalmente en peroxisomas, lisosomas y mitocondrias), lo cual lo convierte en agua y oxígeno molecular. En citosol, la glutatión peroxidasa (GSHPx) provee un mecanismo de protección efectivo, utilizando glutatión reducido (GHS) y convirtiéndolo en glutatión oxidado (GSSG). Este último es reducido nuevamente por la glutatión reductasa (GR).
En varios trabajos se han estudiado los niveles de oxidantes y antioxidantes en pacientes con esquizofrenia. Herken et al, encontraron un incremento en la actividad de las enzimas antioxidantes CAT y GSHPx y mayores niveles de productos de peroxidación de lípidos en pacientes con esquizofrenia, comparados con el grupo control. En este estudio, la actividad de SOD no mostró diferencia entre ambos grupos. ${ }^{20}$ Mahadik et al, también determinaron un incremento en los productos de peroxidación de lípidos en el plasma de pacientes que se encontraban en el primer episodio de psicosis y que no habían recibido medicación. ${ }^{21}$

Los niveles de NO se han visto incrementados en eritrocitos de pacientes con esquizofrenia. ${ }^{22}$ Akyol et al, observaron mayores niveles de productos de peroxidación de lípidos y de NO en plasma, en pacientes con esquizofrenia comparados con el grupo control. También observaron una disminución en la actividad de SOD y un aumento en la de xantinas oxidasas (XO), en los pacientes con esquizofrenia. La actividad de la enzima GSHPx no mostró diferencia entre ambos grupos. ${ }^{15}$ Los resultados contradictorios con respecto a la actividad de algunas de estas enzimas podría deberse al tratamiento con neurolépticos, ya que los cambios en la generación de ROS inducidos por estas drogas pueden afectar las actividades de las enzimas antioxidantes. ${ }^{15}$

Michel et al, determinaron las concentraciones de $\mathrm{Cu}$, $\mathrm{Zn}-\mathrm{SOD}$ y MnSOD en varias áreas corticales (frontal, parietal, temporal y corteza occipital) y subcorticales (putamen, núcleo caudado, tálamo y sustancia innominada) en cerebros postmortem de pacientes con esquizofrenia y controles. Las enzimas $\mathrm{Cu}, \mathrm{Zn}-\mathrm{y}$ MnSOD se vieron significativamente aumentadas en la corteza frontal $y$ sustancia innominada, respectivamente en el grupo índice. No se observaron cambios en las otras áreas. Esto sugiere una relación entre un patrón específico en la distribución neuroanatómica de los procesos de estrés oxidativo y la fisiopatología de la esquizofrenia. ${ }^{23}$ Según los autores tales resultados no resuelven la interrogante de si los cambios en los niveles de estas enzimas son causa primaria en el desarrollo de la esquizofrenia o son cambios secundarios en respuesta al incremento en el estrés oxidativo. Sin embargo, se deben tomar en consideración los efectos de los antipsicóticos como posible fuente de radicales libres. Además, SOD es esencial en el crecimiento celular y diferenciación, por lo que cualquier deterioro de la enzima en el cerebro en desarrollo puede contribuir a alteraciones en la plasticidad neuronal, lo cual puede ser relevante para la manifestación de la esquizofrenia.

Los antioxidantes no enzimáticos también han sido evaluados en pacientes con esquizofrenia. Los niveles en plasma de albúmina, bilirrubina ${ }^{24}$ y ácido úrico ${ }^{25}$ se observaron disminuidos en probandos esquizofrénicos. Suboticanec et al, encontraron que los niveles de ácido ascórbico, en plasma y en orina, de pacientes con esquizofrenia crónica, se encuentran disminuidos al compararlos con los de individuos sanos. ${ }^{26}$ Después de dar 
suplementos de ácido ascórbico durante un mes, las diferencias entre los grupos no fueron significativas, lo cual sugiere que los requerimientos de ácido ascórbico son mayores en pacientes con esquizofrenia. ${ }^{24}$

En pacientes esquizofrénicos se encontraron bajos niveles de $\alpha$-tocoferol comparados con un grupo control. Específicamente aquellos pacientes más afectados, mostraron niveles más bajos. ${ }^{27}$

Los niveles de GSH se han visto disminuidos en tejido postmortem del estriado de esquizofrénicos, comparados con no afectados. ${ }^{28}$ Las enzimas glutamato cisteína ligasa (GCL) y la GSS (glutatión sintetasa) son responsables de la síntesis de GSH. La GCL, está compuesta por dos subunidades: una modificadora (GCLM) y una catalítica (GCLC). Tosic et al, encontraron asociación entre la esquizofrenia y variantes del gen que codifica para la GCLM. ${ }^{29}$ Esta evidencia del papel del gen GCLM en la esquizofrenia es apoyada por otro estudio en el cual se reportó baja expresión de la enzima en fibroblastos de pacientes esquizofrénicos, y actividad disminuida al someterse a estrés oxidativo. ${ }^{30}$ Estos resultados son consistentes con la idea de que el metabolismo anormal de GHS es un factor de riesgo para la esquizofrenia. ${ }^{29}$

Algunos trabajos muestran evidencia de disfunción mitocondrial en la esquizofrenia. La actividad de la citocromo c oxidasa (complejo IV), una enzima clave en la cadena de transporte de electrones, se vio disminuida en la corteza frontal y núcleo caudado de pacientes con esquizofrenia. ${ }^{31}$ En otro estudio se encontró una disminución en los niveles de ARNm y proteínas de las subunidades de $24 \mathrm{kDa}$ y $51 \mathrm{kDa}$ del complejo mitocondrial I, en la corteza prefrontal de pacientes con esquizofrenia comparados con controles sanos. Por otro lado, en la corteza ventral parietoccipital se observó un incremento de los mismos ARNm y proteínas. ${ }^{32}$

Este complejo tiene un papel importante en el control de la fosforilación oxidativa. Por lo tanto, la actividad anormal en este complejo puede conllevar a una disfunción mitocondrial, lo cual puede causar alteraciones en el metabolismo y función neuronal, así como en la plasticidad neuronal y el circuito cerebral, conduciendo a anormalidades en el comportamiento y déficit cognitivo. ${ }^{32}$

También se han estudiado polimorfismos en los genes que codifican para enzimas antioxidantes, debido a que estos podrían conllevar a una predisposición para la esquizofrenia. Akyol y otros autores estudiaron el polimorfismo Ala-9Val del gen $M n S O D$ (ubicado en 6q25) en 153 pacientes esquizofrénicos y 196 controles sanos. Los resultados mostraron diferencias significativas en la distribución genotípica en pacientes (9.2\% Ala/Ala, 69,3\% Ala/Val, $21,6 \% \mathrm{Val} / \mathrm{Val})$ y controles $(23,5 \% \mathrm{Ala} / \mathrm{Ala}, 42,3 \% \mathrm{Ala} / \mathrm{Val}$, $34,2 \% \mathrm{Val} / \mathrm{Val})$, lo cual sugiere que este polimorfismo puede estar relacionado con la fisiopatología de la esquizofrenia. ${ }^{33}$ En otro trabajo se analizó el polimorfismo Pro-197Leu del gen de la glutatión peroxidasa humana ( $G P X 1)$, ubicado en 3 p21.3, en 113 familias nucleares. En este estudio no se encontró desequilibrio de transmisión significativo del polimorfismo, asociado con la presencia de la esquizofrenia en esas familias, lo que podría deberse al reducido tamaño de la muestra. ${ }^{34}$

\section{Estudios sobre esquizofrenia en Costa Rica}

La genética de la esquizofrenia se estudia en Costa Rica desde 1997, en la Universidad de Costa Rica. Las estrategias incluyen un estudio de ligamiento clásico con una familia multigeneracional y varios miembros afectados, un estudio de parejas de hermanos afectados con la enfermedad y un estudio poblacional de asociación. ${ }^{6}$

Los resultados de estos estudios han mostrado evidencia de posible asociación en los cromosomas $8 p$, $13 q$ y $18 p .^{35-37}$ Esto no concuerda con otro estudio realizado por DeLisi et $a l$, en el cual se encontró que 6 regiones de los cromosomas $1 \mathrm{p}, 2 \mathrm{p}, 2 \mathrm{q}, 5 \mathrm{q}, 8 \mathrm{p}$ y $14 \mathrm{q}$ sugieren ligamiento. ${ }^{38}$ Es posible que esto se deba a diferencias entre ambos estudios con respecto a los criterios de inclusión, las pautas diagnósticas o los análisis genealógicos. ${ }^{6}$

Cooper-Casey et al, estudiaron a una familia del Valle Central de Costa Rica compuesta por 28 personas (11 afectados), que posee una herencia parecida a la mendeliana para la esquizofrenia. En este trabajo se encontraron varios marcadores del cromosoma 5 p que aparentemente cosegregan con la enfermedad. El máximo valor de Lod-score fue de 2,70 y lo obtuvo el marcador D5S426. ${ }^{39}$

Walss-Bass et al, realizaron análisis de desequilibrio de ligamiento del cromosoma 18 en familias con pacientes esquizofrénicos, descendientes de la población fundadora del Valle Central de Costa Rica, con apellidos bilineales de origen español. En este trabajo se encontró evidencia de asociación de las regiones 18p11.31 en D18S63, 18q12.3 en D18S474 y 18q22.3ter-qter en D18S70 con la esquizofrenia. ${ }^{40}$

En otro estudio encontramos evidencia de asociación con la esquizofrenia en 4 regiones del cromosoma 8 (8p23.1, 8p21.3, 8q13.3 y 8q24.3), en familias descendientes de la población fundadora del Valle Central de Costa Rica. La evidencia más fuerte de asociación con la enfermedad se presentó en la región 8p23.1, en la cual 3 marcadores (D8S1825, D8S542 y D8S503) dieron resultados positivos. El microsatélite D8S542 mostró la evidencia de asociación más fuerte con el fenotipo esquizofrenia. ${ }^{41}$ Este marcador se encuentra dentro del gen metionina sulfóxido reductasa $\mathrm{A}$ (MsrA), cuyo producto, como se mencionó, es una enzima involucrada en la reparación del daño oxidativo ${ }^{17}$ y que tiene altos niveles de expresión en neuronas y en todo el sistema nervioso. ${ }^{42}$ Por lo anterior, estamos realizando estudios posteriores en este gen para determinar su posible participación en la susceptibilidad a la esquizofrenia en la población costarricense. 


\section{Conclusiones}

Los radicales libres son especies químicas altamente reactivas, generadas durante procesos normales del metabolismo. Una parte de estos cumplen funciones fisiológicas, lo cual es el resultado de un balance entre su producción y el sistema de defensa antioxidante. ${ }^{13}$ Cuando se producen en exceso o el sistema de defensa es deficiente, pueden provocar daño celular. ${ }^{11}$

Existe evidencia de que este balance está alterado en la esquizofrenia. ${ }^{11}$ Debido a que los oxi-radicales pueden destruir los ácidos grasos polinsaturados y por lo tanto influenciar la calidad y cantidad de lípidos de membrana en la esquizofrenia, estos pueden alterar la transducción de señales mediada por receptores de neurotransmisores $\mathrm{y}$, consecuentemente, el procesamiento de información en el cerebro. ${ }^{11}$

La esquizofrenia es una enfermedad compleja, la cual se manifiesta por el efecto aditivo de variantes de muchos genes y factores ambientales. ${ }^{43} \mathrm{El}$ genotipo heredado no conlleva necesariamente al desarrollo de la enfermedad, sino a una mayor susceptibilidad. ${ }^{44}$

Polimorfismos en genes involucrados en el estrés oxidativo pueden aumentar el riesgo de manifestar la enfermedad. Por lo tanto, es de interés estudiar genes como el $M s r A$, que además de sus funciones ${ }^{12}$ posee alta expresión en neuronas ${ }^{42}$ y se encuentra en una región que ha dado evidencia de asociación con la esquizofrenia en Costa Rica. ${ }^{41}$

El control del estrés oxidativo puede contribuir a un menor deterioro en el curso de la enfermedad. Debido a que los antipsicóticos son por sí mismos una posible fuente de radicales libres, es importante una alimentación con buena cantidad de antioxidantes y reducida en calorías. ${ }^{11}$ Además, el excesivo consumo de alcohol y cigarrillos, que es común en pacientes con esquizofrenia y otros trastornos mentales puede aumentar la formación de radicales libres. ${ }^{18}$

Los efectos adversos del estrés oxidativo no son específicos para las enfermedades psiquiátricas y han sido implicados en varias enfermedades como Parkinson, Alzheimer, diabetes mellitus insulino-independiente y esclerosis amiotrófica lateral. Esto sugiere la posibilidad de que el daño producido por los radicales libres no tiene un papel causal en estas enfermedades y que más bien modifica de manera adversa el curso y desenlace de estas. ${ }^{11}$

\section{Referencias}

1. Sawa N, Snyder S. Schizophrenia: diverse approaches to complex disease. Science 2002; 296: 692-695.

2. American Psychiatric Association. Diagnostic and Statistical Manual of Mental Disorders. 4ed., Washington DC: Am Psychiatr Assoc, 1994.

3. McGuffin P, Owen M, Farmer A. Genetic basis of schizophrenia. Lancet 1995; 346: 678-682.

4. Cardno A, Gottesman I. Twin studies of schizophrenia: from bowand-arrow concordances to star wars $\mathrm{Mx}$ and functional genomics. Am J Med Genet 2000; 97: 12-17.

5. Leask SJ. Enviromental influences in schizophrenia: the known and unknown. Adv Psych Treat. 2004;10:323-330.

6. Pacheco A, Raventós H. Genética de la esquizofrenia: avances en el estudio de genes candidatos. Rev Biol Trop 2004; 52: 1-7.

7. Arseneault L, Cannon M, Poulton R, Murray R, Caspi A, Moffit T. Cannabis use in adolescence and risk for adult psychosis: longitudinal prospective study. BMJ 2002; 325: 1212-1213.

8. Owen M, Craddock N, O’Donovan M. Schizophrenia: genes at last? Trends Genet 2005; 21: 518-525.

9. Bardner J, Gershon E. Meta-analysis of whole-genome linkage scans of bipolar disorder and schizophrenia. Molec Psychiatry 2002; 7: 405411.

10. Lewis C, Levinson D, Wise L, DeLisi L, Straub R, Hovatta I, et al. Genome scan meta-analysis of schizophrenia and bipolar disorder, part II: schizophrenia. Am J Hum Genet 2003; 73: 34-48.

11. Mahadik S, Scheffer R. Oxidative injury and potential use of antioxidants in schizophrenia. Prostagland, Leukotr and Essenc Fat Ac 1996; 55: 45-54.

12. Mahadik S, Mukherjee S. Free radical pathology and antioxidant defense in schizophrenia: a review. Schizophr Res 1996; 19: 1-17.

13. Mary J, Vougier S, Picot C, Perichon M, Petropoulos I, Friguet B. Enzymatic reactions envolved in repair of oxidized proteins. Exp Gerontology 2004; 39: 1117-1123.

14. Yao J, Reddy R, van Kammen D. Oxidative damage and schizophrenia: an overview of the evidence and its therapeutic implications. CNS Drugs 2001; 15: 287-310.

15. Akyol Ö, Herken H, Uz E, Fadillioğlu E, Unal S, Söğüt S, et al. The indices of endogenous oxidative and antioxidative processes in plasma from schizophrenic patients. The possible role of oxidant/antioxidant imbalance. Prog in Neuro-Psychoph and Biol Psychiatr 2002; 26: 995-1005.

16. Liou YJ, Lai IC, Lin MW, Bai YM, Lin CC, Liao DL, et al. Haplotype analysis of endothelial nitric oxide synthase (NOS3) genetic variants and tardive dyskinesia in patients with schizophrenia. Pharmacogenet and Genom 2006; 16: 151-157.

17. De Luca A, Sacchetta P, Di Ilio C, Favaloro B. Identification and analysis of the promoter region of the human methionine sulphoxide reductase A gene. Biochem J 2006; 393: 321-329.

18. Floyd R. Antioxidants, oxidative stress and degenerative neurological disorders. PSEBM 1999; 222: 236-245.

19. Hoffer A, Osmond H, Smythies J. Schizophrenia: a new approach. J Ment Sci 1954; 100: 29-35.

20. Herken H, Uz E, Özyurt H, Sögüt S, Virit O, Akyol Ö. Evidence that the activities of erythrocyte free radical scavenging enzymes and the products of lipid peroxidation are increase in different forms of schizophrenia. Molec Psychiatry 2001a; 6: 66-73.

21. Mahadik S, Mukherjee S, Scheffer R, Correnti E, Mahadik J. Elevated plasma lipid peroxides at the onset of nonaffective psychosis. Biol Psychiatry 1998; 43: 674-679. 
22. Herken H, Uz E, Özyurt H, Söğüt S, Virit O, Akyol Ö. Red blood cell nitric oxide levels in patients with schizophrenia. Schizophr Res 2001b; 52: 289-290.

23. Michel T, Thome J, Martin D, Nara K, Zwerina S, Tatschner T, et al. $\mathrm{Cu}, \mathrm{Zn}$ - and $\mathrm{Mn}$-superoxide dismutase levels in brains of patients with schizophrenic psychosis. J Neural Transm 2004; 111: 1191-1201.

24. Yao J, Reddy R, van Kammen D. Abnormal age-related changes of plasma antioxidant proteins in schizophrenia. Psychiatry Res 2000; 97: $137-151$

25. Yao J, Reddy R, van Kammen D. Reduced level of plasma antioxidant uric acid in schizophrenia. Psychiatry Res 1998; 80: 29-39.

26. Suboticanec K, Folnegovic-Smalc V, Korbar M, Mestrovic B, Buzina R. Vitamin C status in chronic schizophrenia. Biol Psychiatry 1990; 28: 959-966.

27. McCreadie R, McDonald E, Wiles D, Campbell G, Paterson J. The Nithsdale Schizophrenia Surveys. XIV: plasma lipid peroxide and serum vitamin $E$ levels in patients with and without tardive dyskinesia, and normal subjects. Br J Psychiatry 1995; 167: 610-617.

28. Yao J, Reddy R, van Kammen D. Altered glutathione redox state in schizophrenia. Dis Mark 2006; 22: 83-93.

29. Tosic M, Ott J, Barral S, Bovet P, Deppen P, Gheorghita F, et al. Schizophrenia and oxidative stress: glutamate cysteine ligase modifier as a susceptibility gene. Am J Hum Genet 2006; 79: 586-592.

30. Gysin R, Tosic M, Chappuis C, Deppen P, Ruiz V, Bovet P, et al Dysregulation of glutamate cysteine ligase in schizophrenia. Paper presented at the $36^{\text {th }}$ Annual Meeting of the Society of Neuroscience, Washington DC, November 12-16 2005.

31. Cavelier L, Jazin E, Eriksson I, Prince J, Bave U, Oreland L, et al Decreased cytochrome c oxidase activity and lack of age related accumulation of mtDNA in brain of schizophrenics. Genomics 1995; 29: $217-228$

32. Karry R, Klein E, Shachar D. Mitochondrial complex I subunits expression is altered in schizophrenia: a postmortem study. Biol Psychiatry 2004; 55: 676-684.

33. Akyol Ö, Yanik M, Elyas H, Namli M, Canatan H, Akin H, et al Association between Ala-9Val polymorphism of Mn-Sod gene and schizophrenia. Progress in Neuro-Psychopharmacology and Biol Psychiatry 2005; 29: 123-131.
34. Shinkai T, De Luca V, Zai G, Shaikh S, Matsumoto C, Arnold P, et al. No association between the Pro197Leu polymorphism in the glutathione peroxidase (GPX1) gene and schizophrenia. Psychiatr Genet 2004; 14: 177-180

35. Balderas T, Montero A, Benavides E, Rodríguez S, Almasy L, Raventós $\mathrm{H}$, et al. Linkage disequilibrium analysis of schizophrenia in the Costa Rican population: preliminary findings on chromosome 18 . Am J Med Genet 2001; 105: 599

36. Escamilla M, Raventos H, Almasy L, Montero P, Balderas T, Rodríguez S, et al. Linkage disequilibrium analysis of schizophrenia and schizoaffective disorder in the Costa Rican population: preliminary findings on chromosome 13. Am J Med Genet 2001; 105: 599.

37. Walss-Bass C, Escamilla MA, Raventós H, Montero A, Armas R, Dassori A, et al. Genome Wide linkage disequilibrium analysis of schizophrenia in the Costa Rican population: preliminary findings on chromosomes 1, 8, 13 y 22. Am J Med Genet 2002; 114: 877.

38. De Lisi L, Mesen A, Rodríguez C, Bertheau A, LaPrade B, Llach M, et al. Genome-wide scan for linkage to schizophrenia in a Spanishorigin cohort from Costa Rica. Am J Med Genet 2002; 114: 497-508.

39. Cooper-Casey K, Mésen-Fainardi A, Galke-Rollins B, Llach M, Laprade B, Rodríguez C, et al. Suggestive linkage of schizophrenia to 5p13 in Costa Rica. Molec Psychiatry 2005; 10: 651- 656.

40. Walss-Bass C, Escamilla MA, Raventós H, Montero A, Armas R, Dassori A, et al. Evidence of genetic overlap of schizophrenia and bipolar disorder: linkage disequilibrium analysis of chromosome 18 in the Costa Rican population. Am J Med Genet 2005; 139: 54-60.

41. Walss-Bass C, Montero AP, Armas R, Dassori A, Contreras S, Liu W, et al. Linkage disequilibrium analices in the Costa Rican population suggests discrete gene loci for schizophrenia at 8p23.1 and 8q13.3. Psychiatr Genet 2006; 16: 159-168.

42. Moskovitz J, Jenkins N, Gilbert D, Copeland N, Jursky F, Weissbach $\mathrm{H}$, et al. Chromosomal localization of the mammalian peptidemethionine sulfoxide reductase gene and its differential expression in various tissues. PNAS 1996; 93: 3205-3208.

43. Mowry B, Nacarrow D. Molecular genetics of schizophrenia. Clin Exp Pharmacol Physiol 2001; 28: 66-69.

44. Dawson E, Murray R. Schizophrenia: a gene at 6p? Curr Biol 1996; 6: 268-271. 